\title{
Posterior Cricoid Region Fluoroscopic Findings: The Posterior Cricoid Plication
}

\author{
Jacqui E. Allen • Cheryl J. White • \\ Rebecca J. Leonard • Peter C. Belafsky
}

Received: 13 May 2010/Accepted: 21 August 2010/Published online: 5 September 2010

(C) The Author(s) 2010. This article is published with open access at Springerlink.com

\begin{abstract}
The region posterior to the cricoid cartilage is challenging to assess fluoroscopically. The purpose of this investigation is to critically evaluate the posterior cricoid (PC) region on fluoroscopy and describe patterns of common findings. This was a case control study. All fluoroscopic swallowing studies performed between June 16, 2009, and February 9, 2010, were reviewed for features seen in the PC region. These findings were categorized into distinct patterns and compared to fluoroscopic studies performed in a cohort of normal volunteers. Two hundred patient studies and 149 healthy volunteer studies were reviewed. The mean age of the referred patient cohort and the volunteer cohort was 57 years $( \pm 19)$ and 61 years $( \pm 16)$, respectively $(p>0.05)$. The patient cohort was 53\% male and the control cohort was $56 \%$ female $(p>0.05)$. Four groups were identified. Pharyngoesophageal webs were seen in 7\% (10/149) of controls and 14\% (28/200) of patients $(p=0.03)$. A PC arch impression was seen in $16 \%$ of patients $(32 / 200)$ and controls $(24 / 149)(p=1)$. A PC plication was demonstrated in 23\% (34/149) of controls and $30 \%(60 / 200)$ of patients $(p=0.13)$. No distinctive PC region findings were seen in 54\% (81/149) of controls and $42 \%(84 / 200)$ of referred patients $(p=0.02)$. Four patients (2\%) had both a web and a PC plication. Four categories of $\mathrm{PC}$ region findings were identified (unremarkable PC region, web, PC arch impression, and PC plication). Both patients referred for swallowing studies and healthy volunteers demonstrated esophageal webs, PC arch impressions,
\end{abstract}

J. E. Allen $(\bowtie) \cdot$ C. J. White · R. J. Leonard · P. C. Belafsky Centre for Voice and Swallowing, University of California, Davis Medical Centre, 2521 Stockton Blvd., Suite 7200,

Sacramento, CA 95817, USA

e-mail: jacquieallen@yahoo.com;

Jacqueline.Allen@ucdmc.ucdavis.edu and PC plications. Only webs were more common in patients than in control subjects $(p=0.03)$. The PC impression and PC plication are likely to represent normal variants that may be identified on fluoroscopic swallow studies.

Keywords Posterior cricoid region .

Fluoroscopic swallowing study - Post-cricoid impression · Posterior cricoid plications - Cricoid arch - Deglutition . Deglutition disorders

Fluoroscopic swallowing studies of the upper aerodigestive tract are an invaluable method of assessing form and function of the oral cavity, oropharynx, hypopharynx, and pharyngoesophageal segment. As a dynamic procedure, information may be obtained regarding both anatomy and coordination of relevant segments. Timing measures are readily obtained and may be compared to well-established normative data to give accurate objective information $[1,2]$. Some regions, however, are more difficult to assess. We define the posterior cricoid (PC) region as the area immediately adjacent to the posterior rim of the cricoid cartilage on the anterior wall of the esophageal inlet. The PC region is somewhat challenging to evaluate on fluoroscopy because of the rapid transit of contrast, its short length and tonic sphincteric closure, and difficulty in correlating fluoroscopic findings with endoscopic anatomy.

Well-documented findings on fluoroscopic swallowing evaluations include hypopharyngeal diverticula, cricopharyngeal bars, and posterior pharyngeal wall abnormalities. Little has been written in regard to PC findings. In 1965, Pitman and Fraser [3] were of the first to note a "postcricoid impression" on barium swallow, which they attributed to a submucosal venous plexus. Since that time, very little 
has been written about PC findings on fluoroscopy. Modern techniques now give us better definition, clarity, and ability to analyze digital images frame by frame. More than 400 fluoroscopic swallowing evaluations are performed in our institution each year. These are reviewed in detail by experienced clinicians, subjectively and objectively analyzed, and then further reviewed by a multidisciplinary dysphagia panel. The purpose of this investigation was to evaluate critically and categorize fluoroscopic findings of the PC region.

\section{Materials and Methods}

Permission to conduct this study was obtained by the institutional review board of the university. All fluoroscopic swallow studies performed at the Center for Voice and Swallowing between June 16, 2009 and February 9, 2010 were reviewed. The features apparent in the PC region were documented. Patient demographics and referral indication were also recorded. Exclusion criteria were age less than 18 years and previous total laryngectomy where the cricoid cartilage was removed. All other studies were included and analyzed on digital media.

One hundred forty-nine dynamic swallowing studies (DSSs) performed in a group of volunteers without dysphagia, neurologic disease, or previous pharyngolaryngeal surgical intervention were deemed a control group and analyzed for comparison.

DSSs were performed using a properly collimated OEC Medical Systems 9800 Radiographic/Fluoroscopic unit that provided a $63 \mathrm{kV}, 1.2 \mathrm{~mA}$-type output for the full field of view mode (12-in. input phosphor diameter). In accordance with our standard protocol, a metal ring of known diameter was taped to the chin or shoulder of the patient. Lateral views were obtained while the patient, seated in an examination chair, was administered liquid barium (EZpaque barium sulfate suspension, $60 \%$ w/v, EZ-EM, Inc., Westbury, NY) boluses of 1,3 , and $20 \mathrm{cc}$ and a 3 -cc paste bolus (EZ-paste, EZ-EM, Inc.) measured with a syringe or graduated cup. The patient was then turned to obtain anteroposterior views and administered liquid barium boluses of 3 and $20 \mathrm{cc}$. Studies were recorded on a Sony MD-1000 DVD recorder (Sony Corp. America, New York, NY) and were played back using WinDVD7 (Intervideo, Corel Corp., Ottawa, ON, Canada). Measures were obtained from digitized images using ImageJ software (National Institutes of Health, Bethesda, MD) and software tools from Iconico, Inc. (New York, NY). Specific measurement techniques have been previously described in detail [1]. An experienced clinician reviewed all studies blind to patient history, treatment, and data analysis. The lateral fluoroscopic view was used to assess the PC region.
Findings were recorded in an Excel spreadsheet and analyzed using the $\chi^{2}$ test of association, odds ratio, Pearson coefficient, and Fisher's exact test. Significance levels were set at $\alpha=0.05$.

\section{Results}

Subjects

Two hundred consecutive subjects who presented for dynamic swallow study were evaluated. The average age of the cohort was 61 years $( \pm 16)$. Fifty-three percent were male. Most common referral indications for DSS were solidfood dysphagia $(65.5 \%, 131 / 200)$, aspiration, cough, or choking $(33.5 \%, 67 / 200)$, and globus or gastroesophageal reflux $(17 \%, 34 / 200)$. Many subjects reported more than one symptom. Underlying disorders included neurological disease (cerebrovascular accident, amyotrophic lateral sclerosis, myasthenia gravis) in $12.5 \%$ (25/200), treatment for head and neck cancer in $12 \%$ (24/200), spinal surgery in 5\% $(10 / 200)$, and autoimmune disease in $2 \%(4 / 200)$.

\section{Control Group}

One hundred forty-nine DSSs performed in healthy volunteers were examined. The mean age of the cohort was 57 years $( \pm 19)$. Fifty-six percent was female. No volunteer reported dysphagia, reflux symptoms, medication to treat reflux, or previous laryngopharyngeal surgery. There was no difference in age or gender between patients and normal volunteers $(p>0.05)$.

\section{Findings}

Analysis of the PC region demonstrated no notable findings in 54\% (81/149) of control subjects and $42 \%(84 / 200)$ of referred subjects $[p=0.02$, odds ratio $(\mathrm{OR})=1.65,95 \%$ $\mathrm{CI}=1.1-2.5]$. The remaining $\mathrm{PC}$ region findings were classified into three types.

\section{Webs}

Pharyngoesophageal webs were seen in $7 \%(10 / 149)$ of controls and $14 \%(28 / 200)$ of referred subjects $(p=0.03$, $\mathrm{OR}=0.44,95 \% \mathrm{CI}=0.2-0.9$ ). Webs were the only PC finding identified significantly more frequently in patients than in control subjects. The webs were located on the anterior wall of the esophageal inlet dorsal and caudal to the cricoid arch. Webs were thin, did not change in shape, and were typically located at the level of the fifth cervical vertebrae. Fluoroscopic view of a pharyngoesophageal web is demonstrated in Fig. 1. 


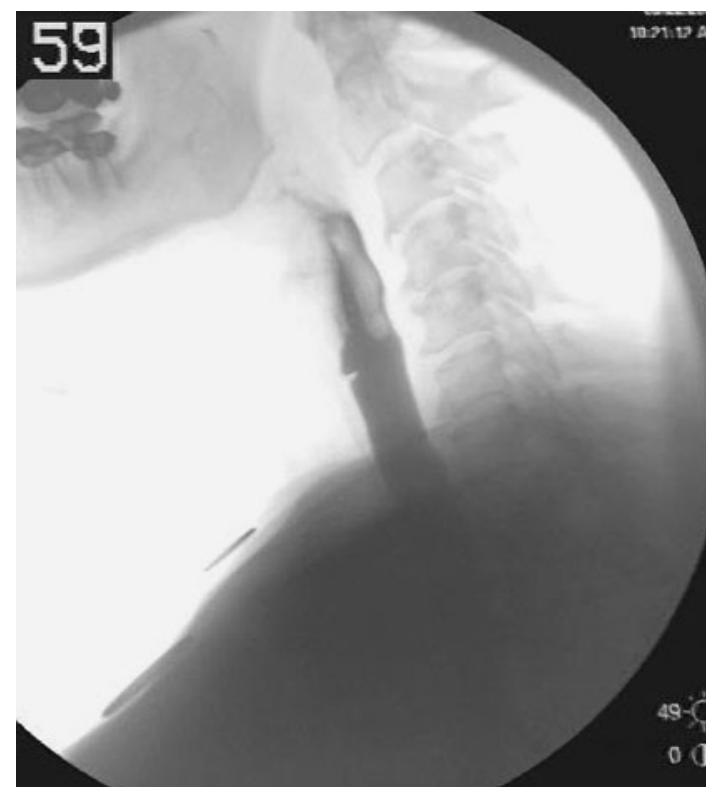

Fig. 1 Fluoroscopic lateral view demonstrating a thin anteriorly based web

Plication

Posterior cricoid plications were seen in $23 \%$ (34/149) of the healthy volunteers and in $30 \%(60 / 200)$ of the referred cohort $(p=0.13, \quad$ OR $=0.69, \quad 95 \% \quad \mathrm{CI}=0.42-1.12)$. The plications were located on the posterior surface of the cricoid arch causing a mobile hyperbolic protrusion into the barium stream that rose with the swallow (Fig. 2). The plication often changed shape during the swallow and

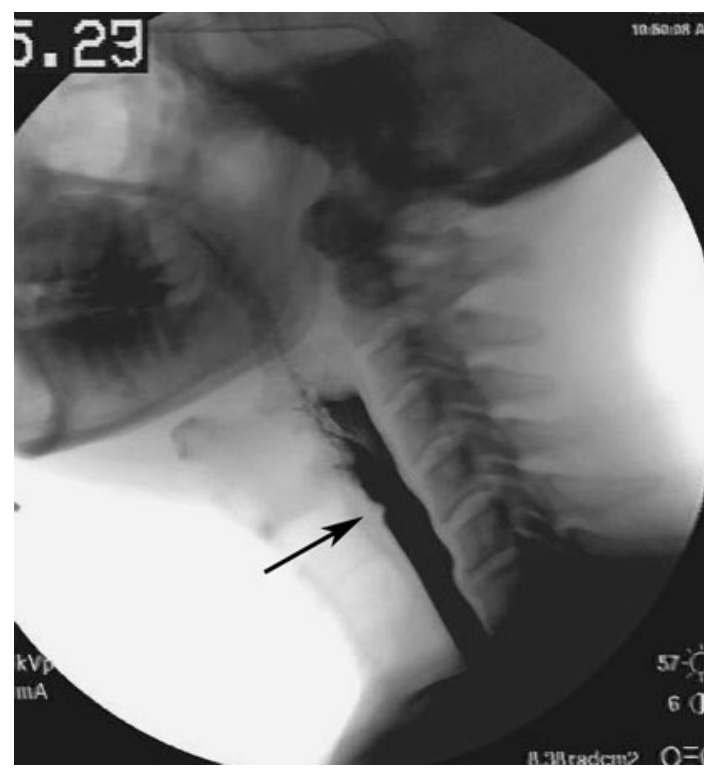

Fig. 2 Fluoroscopic lateral view of posterior cricoid plication (arrow). Note gentle hyperbolic shape with broad base

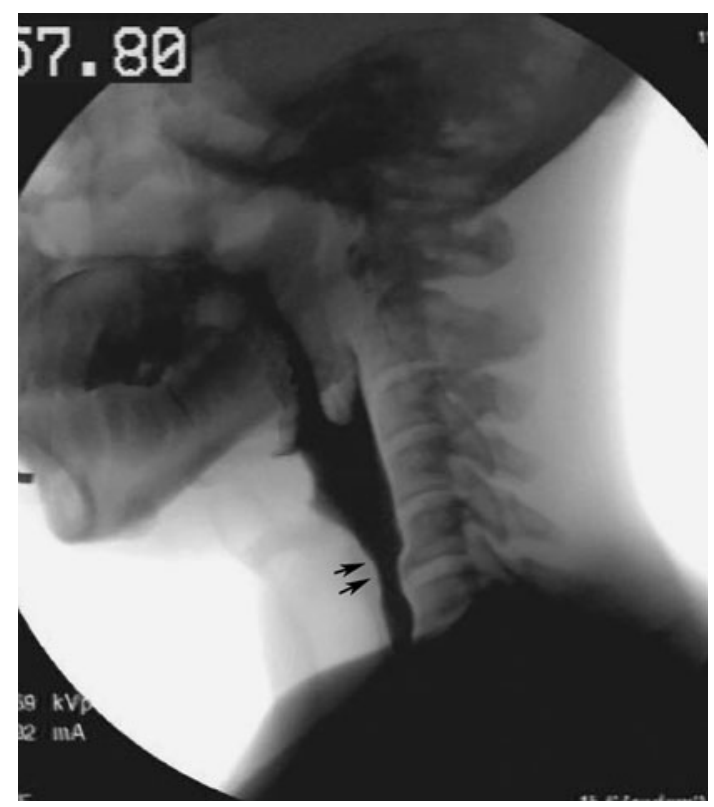

Fig. 3 Fluoroscopic lateral view of posterior cricoid arch impression. Note broad-based flattened appearance at arrows

demonstrated considerable variation in shape between individuals.

\section{Arch Impressions}

Posterior cricoid arch impressions were seen in $16 \%$ $(14 / 149)$ of volunteers and in $16 \%$ (32/200) of referred patients $(p=1, \mathrm{OR}=1,95 \% \mathrm{CI}=0.57-1.8)$. The $\mathrm{PC}$ impressions were located at the posterior rim of the cricoid and appeared to represent an outline of the posterior arch of the cartilage (Fig. 3). They rose with the larynx during swallow but did not change shape during deglutition. Four referred patients (2\%) showed more than one PC finding. In contrast, no control subject demonstrated more than one PC finding.

\section{Discussion}

In 1965, Pitman and Fraser [3] described an impression of the barium stream in the posterior cricoid region in 104 barium studies. They termed this the posterior cricoid (PC) impression and felt that it represented a submucosal venous plexus. Pitman et al. [3, 4] and Butler [5] dissected cadaver larynges after latex injection and described the presence of submucosal bilateral interconnected venous plexuses termed the ventral pharyngolaryngeal venous plexus. They hypothesized that the vessels may dilate and produce a mucosal fold, thus causing the fluoroscopic finding. They felt that the venous plexus was ubiquitous and described it as a normal finding not to be mistaken for a web or 
neoplasm. The finding was reported in $86 \%$ (104/121) of fluoroscopic studies in patients with dysphagia and in $90 \%$ (64/71) of control studies. Pitman and Fraser [3, 4] further described nine subtypes of post-cricoid venous impression and compared them to true webs.

Friedland and Filly [6] supported this description in a case report of a man suspected of having an esophageal tumor based on abnormal fluoroscopic findings. At dissection no mass could be found and they contended that the mass seen represented a PC impression due to a venous plexus as described by Pitman and Fraser [3]. Clements et al. [7] reported fluoroscopic findings of webs, cricopharyngeal bars, and the PC impression in 100 patients with varying gastrointestinal complaints. They found $8 \%$ of the cohort had a web, $18 \%$ had a cricopharyngeal bar, and $71 \%$ had a post-cricoid "defect," which they attributed to lax mucosa in the PC region [7].

Some 25 years after Pitman's description, Dodds et al. [8], in a review of the radiology of normal swallowing, commented on PC region fluoroscopic findings. They noted a PC "impression in the pharyngeal phase of swallowing" and suggested the etiology to be a mucosal plication (rather than a venous plexus) that was a normal variant. Their description lacks mention of the mobility of the structure or its precise location, but it did distinguish this finding from small anterior 1-2-mm cervical webs. Dodds et al. [8] believed these small webs were a common incidental finding and also a variant of normal. Gordon et al. [9] reported esophageal webs as thin projections that did not change with swallow and PC impressions that appeared as "longer, undulating indentation[s]," which they also believed to be due to mucosal folding. They felt that webs may occur in up to $10 \%$ of the general population and that in their cohort, webs were associated with severity of gastroesophageal reflux disease. Ekberg and Nylander [10] reported webs in $12.5 \%$ of 500 dysphagic patients compared to $1.3 \%$ of 150 volunteers seen on contrast swallows.

With more than 400 fluoroscopic studies performed each year at our institution, the recurrent observation of variable PC region features prompted our evaluation and description. With the advantage of modern fluoroscopic imaging, we feel that three distinct groups of PC region findings can be differentiated. Cervical esophageal webs are well documented in the literature [7,9-11]. We observed webs in $7 \%$ of control subjects and $14 \%$ of referred subjects. This is consistent with other reports [9-11]. We feel that a web is a thin eccentric rim of normal esophageal mucosa and submucosa. Webs may be symptomatic or asymptomatic. They can be associated with systemic disease, such as in Plummer-Vinson Syndrome, but most commonly they are isolated findings. In this study there was a statistically higher rate of webs in patients presenting for a DSS compared to asymptomatic controls $(p=0.03)$.
Two other groups with distinct findings were observed. The PC impression was identified in $16 \%$ of volunteers and $16 \%$ of referred patients. We believe that the PC impression represents an outline of the posterior surface of the cartilaginous cricoid arch. It is mobile during deglutition, rising with the larynx, but does not change shape during swallowing. The profile is rather flatter than either a web or a PC plication, in keeping with the anatomy and contour of the PC lamina.

We have termed the final category of PC findings the PC plication. A plication was identified in $23 \%$ of volunteers and $30 \%$ of referred subjects $(p>0.05)$. The indentation in the barium stream caused by the plication is more broadbased than a web but does not correspond to the rim of the cricoid arch, as does a PC arch impression. Plications are hyperbolic in shape and are mobile, consistently seen arising at the level of $\mathrm{C} 5$ and then elevating and descending with the larynx. This is similar to the description of Pitman's "impression" and Dodds "plication" [3, 8]. We believe that this plication is what Pitman and Fraser referred to as a "post cricoid impression." Because it does not correspond to the impression of the cricoid cartilage, we feel that plication is a more appropriate term and that the term "impression" should be reserved for the description of the indentation caused by the cricoid arch. It seems likely that the nine subtypes of PC irregularities described by Pitman and Fraser [3] represent different perspectives of the same structure caught at various moments of the barium passage. This became obvious to us with our ability to view digital images frame by frame, a technology not available to Pitman and Fraser in 1965. Thus, we do not distinguish subtypes of plications but rather classify all of them simply as PC plications.

We feel that the PC plication represents a fold of mucosa possibly overlying slips of muscle that arise from the longitudinal layer of the esophagus and extend up to the midline ridge of the PC arch. A large barium bolus maximally distends the upper esophageal sphincter and can delineate these mucosa-covered fibers. Ekberg and Nylander [10] also felt that maximal distension of the esophagus with a large bolus was crucial in identifying these features. Although we did not examine the association of PC findings and patient symptoms, we believe that the plication should be considered a variant of normal because it appears at similar rates in healthy subjects and referred patients and always appeared small and nonobstructive. The observation that it elevates with the swallow suggests attachment to the hyolaryngeal complex.

The size of our cohort and control groups was large, affording some measure of confidence in the relative prevalence of the PC region fluoroscopic findings described. All patient studies were included except children and postlaryngectomy patients. Therefore, a wide study population is 
represented, which increases the ability to generalize the findings. The Swallowing Centre, however, is a tertiary academic unit and may not be representative of patients seen in general practice. Videofluoroscopic DSSs are a twodimensional representation of a three-dimensional structure: the pharynx. While we have described what can be seen on DSS as being related to the cricoid region, it is possible that superimposition of structures laterally may produce some of these features. Nonetheless, we still feel that the distinction between groups based on fluoroscopy is valid. Direct anatomical correlation would be helpful although difficult to do in subjects, as just introducing instrumentation such as a rigid laryngoscope or esophagoscope can change the shape and features of this region. Endoscopic views are also difficult due to rapid transit of the region by the endoscope, coupled with mucosal collapse around the endoscope, obstructing views. Cadaver studies are limited by fixation artifact and loss of tissue pliability and dynamic tissue reaction.

\section{Conclusion}

Posterior cricoid region findings on fluoroscopic swallow studies can be classified as pharyngoesophageal webs, posterior cricoid plications, and posterior cricoid arch impressions. Webs were identified significantly more often in patients than in control subjects and may be symptomatic or asymptomatic. Posterior cricoid plications and arch impressions occur with similar frequency in patients and volunteers and likely represent variants of normal anatomy.

\section{Conflict of interests None}

Open Access This article is distributed under the terms of the Creative Commons Attribution Noncommercial License which permits any noncommercial use, distribution, and reproduction in any medium, provided the original author(s) and source are credited.

\section{References}

1. McKenzie S, Leonard R. DSS: swallow evaluation with videofluoroscopy. In: Leonard R, Kendall K, editors. Dysphagia assessment and treatment planning: a team approach. San Diego: Plural Publishing, Inc.; 2008. p. 201-6.

2. Kendall K, McKenzie S, Leonard R. Dynamic swallow studyobjective measures and normative data in adults. In: Leonard R, Kendall K, editors. Dysphagia assessment and treatment planning: a team approach. San Diego: Plural Publishing, Inc.; 2008. p. 233-64.

3. Pitman RG, Fraser GM. The post-cricoid impression on the oesophagus. Clin Radiol. 1965;16:34-9.

4. Pitman RG. The postcricoid impression on the esophagus. AJR Am J Roentgenol. 1992;158:690.

5. Butler H. The veins of the esophagus. Thorax. 1951;6:276-96.

6. Friedland GW, Filly R. The postcricoid impression masquerading as an esophageal tumor. Dig Dis. 1975;20:287-91.

7. Clements JL, Cox GW, Torres WE, Weens HS. Cervical esophageal webs-a roentgen-anatomic correlation. AJR Am J Roentgenol. 1974;121:221-31.

8. Dodds WJ, Stewart ET, Logemann JA. Physiology and radiology of the normal oral and pharyngeal phases of swallowing. AJR Am J Roentgenol. 1990;154:953-63.

9. Gordon AR, Levine MS, Redfern RO, Rubesin SE, Laufer I. Cervical esophageal webs: association with gastroesophageal reflux. Abdom Imaging. 2001;26:574-7.

10. Ekberg O, Nylander G. Webs and web-like formations in the pharynx and cervical esophagus. Diagn Imaging. 1983;52:10-8.

11. Nosher JL, Campbell WL, Seaman WB. The clinical significance of cervical esophageal and hypopharyngeal webs. Radiology. 1975; 117:45-7.

Jacqui E. Allen $\mathrm{MBChB}$, FRACS

Cheryl J. White MA, CCC

Rebecca J. Leonard MS, PhD

Peter C. Belafsky $\mathrm{MD}, \mathrm{PhD}$ 